\title{
The Stochastic Bifurcation Behaviour of Speculative Financial Markets*
}

\author{
Carl Chiarella ${ }^{1}$, Xue-Zhong $\mathrm{He}^{1}$, Duo Wang ${ }^{2}$ and Min Zheng ${ }^{1,2}$ \\ ${ }^{1}$ School of Finance and Economics, University of Technology, Sydney \\ PO Box 123, Broadway, NSW 2007, Australia \\ carl.chiarella@uts.edu.au, tony.he1@uts.edu.au,_min.zheng@uts.edu.au \\ ${ }^{2}$ School of Mathematical Sciences, Peking University \\ Beijing 100871, P. R. China \\ dwang@math.pku.edu.cn
}

\begin{abstract}
This paper establishes a continuous-time stochastic asset pricing model in a speculative financial market with fundamentalists and chartists by introducing a noisy fundamental price. By an application of stochastic bifurcation theory, the limiting market equilibrium distribution is examined numerically. It is shown that speculative behaviour of chartists can lead the market price displaying different forms of equilibrium distributions. In particular, when chartists are less active, there is a unique equilibrium distribution which is stable. However, when the chartists become more active, a new equilibrium distribution will be generated and become stable. The corresponding stationary density will change from single peak to crater-like density. The change of stationary distribution is characterized by a bimodal logarithm price distribution and fat tails. The paper demonstrates that stochastic bifurcation theory is a useful tool in providing insights into various types of financial market behaviour in a stochastic environment.
\end{abstract}

Key Words: Heterogeneous agents, speculative behaviour, random dynamical systems, stochastic bifurcations, invariant measures.

\section{Introduction}

The theory of random dynamical systems provides a very powerful mathematical tool for understanding the limiting behaviour of stochastic systems. Recently, it has

${ }^{*}$ Financial support from the Australian Research Council (ARC) under a Discovery Grant (DP0450526), the UTS under a Research Excellence Grant, and the National Science Foundation of China (10571003) are gratefully acknowledged. 
been applied to economics and finance to help in understanding the stochastic nature of financial models with random perturbations. In particular, the study of the limiting distribution of various stochastic models in economics and finance gives a good description of stationary markets. For example, Föllmer et al. [9] study the existence and uniqueness of the limit distribution in a discrete financial market model with different expectations through stochastic learning and Böhm and Chiarella [4] investigate the long-run behaviour (stationary solutions) for mean-variance preferences under various predictors. Those models mainly focus on the existence, uniqueness and stability of limiting distributions of discrete time financial models, rather than the changes of existence and stability of multiple limiting distributions of continuous-time financial models as their parameters change.

Stochastic bifurcation theory has developed to study the changes of existence and stability of limiting distributions. There seems to have been no application of it to heterogeneous agent models of continuous-time financial markets except the one-dimensional continuously randomized version of Zeeman's [15] stock market model studied by Rheinlaender and Steinkamp [12]. These authors show a stochastic stabilization effect and possible sudden trend reversal in the financial market. For higher dimensional financial market models with heterogeneous agents in the continuous-time framework, the application of stochastic bifurcation theory faces many challenges. In this paper, we take the very basic heterogeneous agent financial market model of fundamentalists and chartists developed by Beja and Goldman [3] and Chiarella [5] and set it up as a two-dimensional stochastic model by introducing a noisy fundamental price in a continuous-time framework. We then use stochastic bifurcation theory to analyze numerically the changes and stability of multiple limiting distributions of the two-dimensional financial market model as the chartists' behaviour changes. The numerical analysis of our speculative market model is largely motivated by the work of Arnold et al. [2] and Schenk-Hoppé [14] on the noisy Duffing-van der Pol oscillator. To provide a complete picture of the market equilibrium behaviour of the model as a parameter capturing the chartists behaviour changes, we conduct our analysis from the viewpoints of both dynamical and phenomenological bifurcations. The so-called dynamical (D)-bifurcation examines the evolution of an initial point forward and backward in time and captures all the stochastic dynamics of the SDEs, while the so-called phenomenological (P)-bifurcation studies a stationary measure corresponding to the one-point motion. As indicated in Schenk-Hoppé [14] and the references cited there, the difference between P-bifurcation and D-bifurcation lies in the fact that the P-bifurcation approach focuses on long-run probability distributions, while the D-bifurcation approach is based on the invariant measure, the multiplicative ergodic theorem, the Lyapunov exponents, and the occurrence of new invariant measures. However, the P-bifurcation has the advantage of allowing one to visualize the changes of the stationary density functions. Our results show that, as the chartists change their behaviour (through their extrapolation of price trend), the market price can display different forms of equilibrium distribution. In particular, when chartists are 
less active, the market has a unique equilibrium distribution which is stable. However, when the chartists become more active, a new equilibrium distribution will be generated and become stable whilst the original equilibrium distribution becomes unstable. The corresponding stationary density will change from single peak to crater-like density. The market price can be driven away from the fundamental price. The change of stationary distribution is characterized by a bimodal logarithm price distribution and fat tails.

The structure of the paper is as follows. In Section 2, we first outline the extended model of Beja and Goldman [3] and Chiarella [5] with a stochastic fundamental price. In Sections 3 and 4, the stochastic dynamical behaviour is analyzed from the viewpoints of invariant measures and stationary measures, respectively. The paper is then concluded in Section 5.

\section{The Model}

Consider a financial market which consists of two type investors, fundamentalists and chartists and two types of assets, a risky asset (e.g. a stock market index) and a riskless asset (e.g. a government bond). In the market, the transactions and price adjustments occur simultaneously. The changes of the risky asset price $P(t)$ are brought about by aggregate excess demand of fundamentalists $\left(D_{t}^{f}\right)$ and chartists $\left(D_{t}^{c}\right)$ at a finite speed of price adjustment. Accordingly, these assumptions may be expressed as

$$
d p(t)=\left[D_{t}^{f}+D_{t}^{c}\right] d t
$$

where $p_{t}=\ln P(t)$ is the logarithm of the risky asset price $P(t)$ at time $t$.

The excess demand of the fundamentalists is assumed to depend on the market price deviation from the fundamental price, so that

$$
D_{t}^{f}(p(t))=a[F(t)-p(t)]
$$

where $a>0$ and $F(t)$ denotes the logarithm of the fundamental price that clears fundamental demand at time $t$, that is $D_{t}^{f}(F(t))=0$. For the fundamental price $F(t)$, in accordance with the theory of equilibrium prices in perfect markets, the successive changes in equilibrium values must be statistically independent. This proposition is usually formalized by the statement that $F(t)$ follows a random walk. Using the notation of stochastic differential equations, the fundamental value $F(t)$ can be considered to follow

$$
d F=\sigma \circ d W
$$

where $W$ is a two-sided Wiener process on the Wiener space $(\Omega, \mathcal{F}, \mathbb{P})$ with zero drift and unit variance per unit time and $\sigma>0$ is the instantaneous standard deviation (volatility) of the fundamental returns. Here the circle o indicates that the SDE is in the Stratonovich sense, rather than Itô sense. We use the Stratonovich over Itô SDE 
formalism since it has the advantage of the validity of chain rule without the addition of second derivative terms.

Unlike the fundamentalists, the chartists' excess demand is assumed to reflect the potential for direct speculation on price changes, including the adjustment of the price towards equilibrium. Let $\psi(t)$ denote the chartists' assessment of the current trend in $p(t)$. Both the market price and the fundamental price are assumed to be detrended by the risk-free rate. Correspondingly, we can assume that the risk-free rate is zero. Then the excess demand of chartists is assumed to be given by $D_{t}^{c}(p(t))=h(\psi(t))$, where $h$ is a nonlinear continuous and differentiable function, satisfying $h(0)=0, h^{\prime}(x)>0$ for $x \in$ $\mathbb{R}, \lim _{x \rightarrow \pm \infty} h^{\prime}(x)=0, h^{\prime \prime}(x) x<0$ for $x \neq 0, \sup _{x}|h(x)|<\infty$, and $\sup _{x}\left|h^{\prime}(x)\right|<\infty$.

Note that the chartists' speculation on the adjustment of the log-price toward equilibrium must depend in part on recent log-price changes and will be an adaptive process of trend estimation. One of the simplest assumptions is that $\psi$ is taken as an exponentially declining weighted average of past log-price changes. That is, $\psi(t)=c \int_{-\infty}^{t} e^{-c(t-s)} d p(s)$. This can be expressed as the first order differential equation $d \psi(t)=c[\dot{p}(t)-\psi(t)] d t$, where $c \in(0, \infty)$ is the decay rate, which also measures the speed with which chartists adjust their estimate of the trend to past log-price changes.

Summarizing the above set up, we obtain the asset price dynamics

$$
\left\{\begin{array}{l}
d p(t)=a[F-p(t)] d t+h(\psi(t)) d t \\
d \psi(t)=[-a c p(t)-c \psi(t)+c h(\psi(t))+a c F] d t \\
d F=\sigma \circ d W
\end{array}\right.
$$

When $\sigma=0$, the fundamental price is a constant with $F \equiv F^{*}$. Then the system (2.2) is reduced to the model studied by Beja and Goldman [3] and Chiarella [5]. It has a unique steady-state $(\bar{p}, \psi)=\left(F^{*}, 0\right)$. Performing a local linear analysis around the steady-state, Beja and Goldman [3] show that the steady-state is locally stable if and only if $c<c^{*}=\frac{a}{b-1}$, where $b=h^{\prime}(0)$ represent the slope of the demand function at $\psi=0$ for the chartists. Based on a nonlinear analysis, Chiarella [5] shows that (i) if the chartists' demand is sufficiently low (so that $b<1$ ), then the asset price will converge to the steady-state equilibrium; (ii) if the chartists' demand is large enough (so that $b>1$ ), then the asset price will converge locally to the steady-state equilibrium when the chartists revise their estimate of the trend slowly (so that $c<c^{*}$ ). However, if chartists accelerate the revision of their estimate of the trend sufficiently so that $c$ surpasses $c^{*}$, then a Hopf bifurcation occurs and the instability of the steady-state equilibrium appears with prices converging to a limit cycle. Therefore, complex phenomena start to appear when chartists' demand is sufficient strong (so that $b>1$ ).

When $\sigma \neq 0$, letting $\phi=\frac{d \psi}{d t}$, a nonlinear Stratonovich-SDE system in $\psi$ and $\phi$ can be obtained, namely

$$
\left\{\begin{aligned}
d \psi & =\phi d t \\
d \phi & =\left[-a-c+c h^{\prime}(\psi)\right] \phi d t-a c \psi d t+a c \sigma \circ d W
\end{aligned}\right.
$$


Once the dynamics of $\psi(t)$ have been obtained, the dynamics of the price $p(t)$ can be obtained by integrating the first equation in (2.2).

To clarify the dynamic behaviour of the model (2.3), it is necessary to deal with the random dynamical system that it generates. We refer the reader to Arnold [1] for a more detailed and systematic treatment of the theory of RDSs. A random dynamical system consists of two ingredients: a model describing a dynamical system perturbed by noise and a model of the noise. Here the model of the noise is the standard two-sided Wiener process $\left\{W_{t}\right\}_{t \in \mathbb{R}}$, which is modelled by an ergodic metric dynamical system $\left\{\vartheta_{t}\right\}_{t \in \mathbb{R}}$ on $(\Omega, \mathcal{F}, \mathbb{P})$ with $\vartheta_{t} \omega(s):=\omega(t+s)-\omega(t)$ and $W_{t}(\omega)=\omega(t)$.

A dynamical system perturbed by noise, that is a random dynamical system (RDS), is modelled by the following co-cycle property. A local $C^{\infty}$ random dynamical system on $\mathbb{R}^{2}$ over $\left(\Omega, \mathcal{F}, \mathbb{P},\left(\vartheta_{t}\right)_{t \in \mathbb{R}}\right)$ is defined as a measurable mapping $\varphi: \mathcal{D} \rightarrow \mathbb{R}^{2},(t, \omega, \mathbf{x}) \mapsto$ $\varphi(t, \omega, \mathbf{x})(=: \varphi(t, \omega) \mathbf{x})$, where $\mathcal{D} \in \mathcal{B}(\mathbb{R}) \otimes \mathcal{F} \otimes \mathcal{B}\left(\mathbb{R}^{2}\right)$ and $\mathcal{B}\left(\mathbb{R}^{n}\right)$ is the Borel $\sigma$-algebra generated by $\mathbb{R}^{n}$, such that (i) $\varphi$ is a co-cycle, that is, $\varphi(0, \omega)=i d$, the identity map, and $\varphi(t+s, \omega)=\varphi\left(t, \vartheta_{s} \omega\right) \circ \varphi(s, \omega)$; (ii) $(t, \mathbf{x}) \mapsto \varphi(t, \omega, \mathbf{x})$ is continuous and $\mathbf{x} \mapsto \varphi(t, \omega) \mathbf{x}$ is a $C^{\infty}$ diffeomorphism.

Similar to Schenk-Hoppé [13], the particular structure of (2.3) allows us to show that the solution of $(2.3) \varphi(t, \cdot) \mathbf{x}_{0}$ of $(2.3)$ with the initial value $\mathbf{x}_{0}=\left(\psi_{0}, \phi_{0}\right)$ defines a global random dynamical system, i.e. $\mathcal{D}=\mathbb{R} \times \Omega \times \mathbb{R}^{2}$. Then, in what follows, we study the stochastic dynamical behaviour of the global random dynamical system $\varphi(t, \cdot) \mathbf{x}_{0}$.

In the following two sections, we will see how the introduction of randomness changes the stochastic behaviour significantly from both the dynamical and phenomenological points of view. For the rest of this paper, we only consider the case $b>1$.

\section{D-Bifurcation}

The dynamical or D-bifurcation approach deals with invariant measures, the application of the multiplicative ergodic theorem, and random attractors. In stochastic models, random invariant measures are the corresponding concept for invariant sets in deterministic models. Invariant measures are of fundamental importance for an RDS as they encapsulate its long-run and ergodic behaviour.

For a given RDS $\varphi$ over $\vartheta, \mu \in \mathcal{P} r\left(\Omega \times \mathbb{R}^{2}\right)$, where $\operatorname{Pr}\left(\Omega \times \mathbb{R}^{2}\right)$ is a set of all probability measures in $\left(\Omega \times \mathbb{R}^{2}, \mathcal{F} \otimes \mathcal{B}\left(\mathbb{R}^{2}\right)\right)$, is said to be an invariant measure for the $\operatorname{RDS} \varphi$, if (i) $\pi_{\Omega} \mu=\mathbb{P}$ and (ii) $\Theta(t) \mu=\mu$ for all $t \in \mathbb{R}$, where $\pi_{\Omega} \mu$ stands for the marginal of $\mu$ on $\Omega$ and $\Theta(t):\left(\Omega, \mathbb{R}^{2}\right) \rightarrow\left(\Omega, \mathbb{R}^{2}\right),(\omega, \mathbf{x}) \mapsto(\vartheta(t) \omega, \varphi(t, \omega) \mathbf{x})=: \Theta(t)(\omega, \mathbf{x})$. Condition (i) indicates that the noise is not at our disposal, which means that the marginal of the invariant measure on the probability space $\Omega$ has to be the given measure $\mathbb{P}$. Condition (ii) implies that the stochastic process $\{\Theta(t)\}$ with the initial distribution $\mu$ has the same distribution $\mu$ at every time $t$, i.e., $\mu$ is invariant for $\{\Theta(t)\}$.

Every measure $\mu \in \operatorname{Pr}\left(\Omega \times \mathbb{R}^{2}\right)$ with marginal $\mathbb{P}$ on $\Omega$ can factorize, that is, there 
exists a $\mathbb{P}$-a.s. unique measurable map $\mu: \Omega \rightarrow \mathcal{P} r\left(\mathbb{R}^{2}\right), \omega \rightarrow \mu_{\omega}$ (probability kernel) such that $\mu(d \omega, d \mathbf{x})=\mu_{\omega}(d \mathbf{x}) \mathbb{P}(d \omega)$. In the following, we identify a measure $\mu$ with its factorization $\left(\mu_{\omega}\right)_{\omega \in \Omega}$. Then, a measure $\mu \in \mathcal{P} r\left(\Omega \times \mathbb{R}^{2}\right)$ is invariant under $\varphi$ if and only if for all $t \in \mathbb{R}, \varphi(t, \omega) \mu_{\omega}=\mu_{\vartheta_{t} \omega}, \mathbb{P}$-a.s..

A D-bifurcation occurs if a reference invariant measure $\mu^{\gamma}$ depending on the parameter $\gamma$ loses its stability at some point $\gamma_{D}$, and another invariant measure $\nu^{\gamma} \neq \mu^{\gamma}$ exists for some $\gamma$ in each neighborhood of $\gamma_{D}$ with $\nu^{\gamma}$ converging weakly to $\mu^{\gamma_{D}}$ as $\gamma \rightarrow \gamma_{D}$. Therefore, the D-bifurcation focuses on the loss of stability of invariant measures and on the occurrence of new invariant measures. The discussion in the following two sections examines these two aspects of the D-bifurcation.

\subsection{Lyapunov Exponents}

Similar to the stability analysis of a deterministic dynamical system, the stability of invariant measures of a random dynamical system is described by the Lyapunov exponents given by the multiplicative ergodic theorem (MET) (see Arnold [1]) which is the stochastic equivalent of the eigenvalue-eigenspace decomposition of deterministic dynamical systems.

As in the deterministic case, we take $c$, the speed of adjustment of the chartist towards the trend, as the bifurcation parameter. Let $\mu^{c}$ be an invariant ergodic probability measure for the random dynamical system $\varphi_{c}$ generated by (2.3) depending on the parameter c. Consider the linearization (variational equation) corresponding to (2.3), namely

$$
\left\{\begin{aligned}
d u & =v d t \\
d v & =\left[c h^{\prime \prime}(\psi) \phi-a c\right] u d t+\left[-a-c+c h^{\prime}(\psi)\right] v d t,
\end{aligned}\right.
$$

where $(\psi, \phi)$ is the solution of $(2.3)$ with initial value $\mathbf{x}$. By the MET, there exists an invariant set $\Gamma_{c} \subset \Omega \times \mathbb{R}^{2}$ with $\mu^{c}\left(\Gamma_{c}\right)=1$ satisfying the conditions: (1)there exist two Lyapunov exponents of the invariant measure $\mu^{c}, \lambda_{1}^{c} \geq \lambda_{2}^{c}$, which are a.e. constants in $\Gamma_{c}$, and (2) for any $(\omega, \mathbf{x}) \in \Gamma_{c}$, there exists an invariant splitting $E_{1}^{c}(\omega, \mathbf{x}) \oplus E_{2}^{c}(\omega, \mathbf{x})=\mathbb{R}^{2}$, such that any solution $V_{t}^{c}(\omega, \mathbf{x})$ with initial value $V_{0}^{c} \neq 0$ of the variational equation (3.1) has the exponential growth rates

$$
\lambda_{i}^{c}=\lim _{t \rightarrow \infty} \frac{1}{t} \log \left\|V_{t}^{c}(\omega, \mathbf{x})\right\| \quad \text { if } \quad V_{0}^{c} \in E_{i}^{c}(\omega, \mathbf{x}), \quad i=1,2 .
$$

Hence the Lyapunov exponent can be said to be the stochastic analogue of "the real part of an eigenvalue" of a deterministic system (at a fixed point) and $E_{1,2}^{c}$ correspond to the eigenspaces. In addition, the stochastic analogue of "the imaginary part of the eigenvalue" is the rotation number $\kappa\left(\mu^{c}\right)$, which is defined as the average phase speed of $V_{t}^{c}(\omega, \mathbf{x})$, that is $\kappa\left(\mu^{c}\right)=\lim _{t \rightarrow \infty} \frac{1}{t} \arg V_{t}^{c}(\omega, \mathbf{x})$. A value of $\kappa\left(\mu^{c}\right) \neq 0$ means that the stochastic flow will, in a fluctuating manner, converge to (diverge from) the attractor (repeller). Therefore the D-bifurcation approach is a natural generalization of deterministic bifurcation theory, if one adopts the viewpoint that an invariant measure is the 
stochastic analogue of an invariant set, for instance a fixed point, and the MET is the stochastic equivalent of linear algebra. For deterministic systems we use linear algebra to determine the stable and unstable eigenspaces. For stochastic systems we use the MET to determine the so-called Oseledets spaces (a concept developed out of the work of Oseledets [10]) as described following (3.1).

To approximate the Lyapunov exponents and rotation number, we use stochastic numerical methods to solve the variational equations (3.1). In the following, we take $h(x)=\alpha \tanh (\beta x)$, where $\alpha, \beta(>0)$ so that $b=h^{\prime}(0)>1$ is satisfied, which corresponds to the appearance of complex phenomena in the deterministic case. The computational scheme proceeds as follows. We first solve the original SDEs (2.3) by the Euler-Maruyama scheme, substitute this solution into the variational equations (3.1), and solve this linear SDE system with the same numerical scheme as for (2.3). Then using the Gram-Schmidt Orthonormalization method (see pages 74-80 in Parker and Chua [11]), we can simultaneously estimate all Lyapunov exponents of a stable invariant measure. In addition, the rotation number can be calculated from the definition at the same time.

To detect the instability of an invariant measure, we calculate the Lyapunov exponents and rotation number for the time reversed SDEs. This means that we make a time transforation (time reversion) $t \rightarrow-t$ and let $\widehat{\psi}(t):=\psi(-t), \widehat{\phi}(t):=\phi(-t)$ and $\widehat{W}(t):=W(-t)$. Then the SDEs $(2.3)$ becomes

$$
\left\{\begin{array}{l}
d \widehat{\psi}(t)=-\widehat{\phi}(t) d t \\
d \widehat{\phi}(t)=\left[a+c-c h^{\prime}(\widehat{\psi}(t))\right] \widehat{\phi}(t) d t+a c \widehat{\psi}(t) d t+a c \sigma \circ d \widehat{W}(t) .
\end{array}\right.
$$

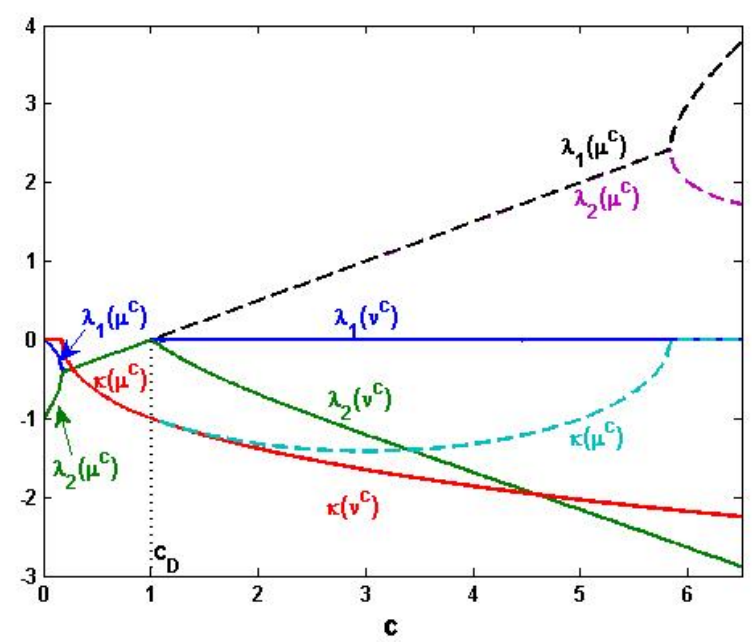

Figure 1: Lyapunov exponents and rotation number as a function of $c$ for $a=1, \alpha=2$, $\beta=1, \sigma=0.02$.

We take $a=1, \alpha=2, \beta=1, \sigma=0.02$ and vary $c$. The iteration time has been taken as 1000 time units with step size 0.001 for each time unit. Fig. 1 shows the Lyapunov exponents of the invariant measures and their rotation numbers, as functions of the parameter $c$. Note that with $c$ increasing to $c_{D} \approx c^{*}=\frac{a}{b-1}$, the Lyapunov exponents change from negative values to positive ones, which means that the stability of the 
reference measure $\mu^{c}$ transfers from stable to unstable. When $\lambda_{1,2}\left(\mu^{c}\right)>0$, we obtain two other Lyapunov exponents, denoted by $\lambda_{1,2}\left(\nu^{c}\right)$, satisfying $\lambda_{2}\left(\nu^{c}\right)<\lambda_{1}\left(\nu^{c}\right) \leq 0$, which indicates the appearance of a new stable invariant measure $\nu^{c}$. This means that, there always exists an invariant measure $\mu^{c}$ in the market. However, when the chartists extrapolate the price trend weakly (so that $c<c_{D}$ ), this invariant measure is unique and stable; when the chartists extrapolate the price trend strongly (so that $c>c_{D}$ ), there exists a new invariant measure $\nu^{c}$ such that the original invariant measure $\mu^{c}$ becomes unstable and the new invariant measure $\nu^{c}$ becomes stable. We note that the bifurcation value $c_{D}$ is very close to the bifurcation value $c^{*}$ for the deterministic case discussed in the previous section. In the deterministic case, a Hopf bifurcation occurs when $c=c^{*}$, leading to the appearance of the limit cycle. In the following section, we characterize the stochastic Hopf bifurcation of the invariant measure by using the concept of random attractors.

\subsection{Random Fixed Points and Random Attractors}

Changes in the Lyapunov exponents indicate the changes of invariant measures. In order to detect the changes of invariant measure, we first consider a random fixed point (i.e. a random variable $\mathbb{x}(\omega)$ satisfying $\varphi(t, \omega) \mathbb{x}(\omega)=\mathbb{x}\left(\vartheta_{t} \omega\right)$ for almost all $\omega \in \Omega$ and $t \in T)$, because a random fixed point corresponds to a random invariant Dirac measure $\delta_{\mathbb{X}(\omega)}$.

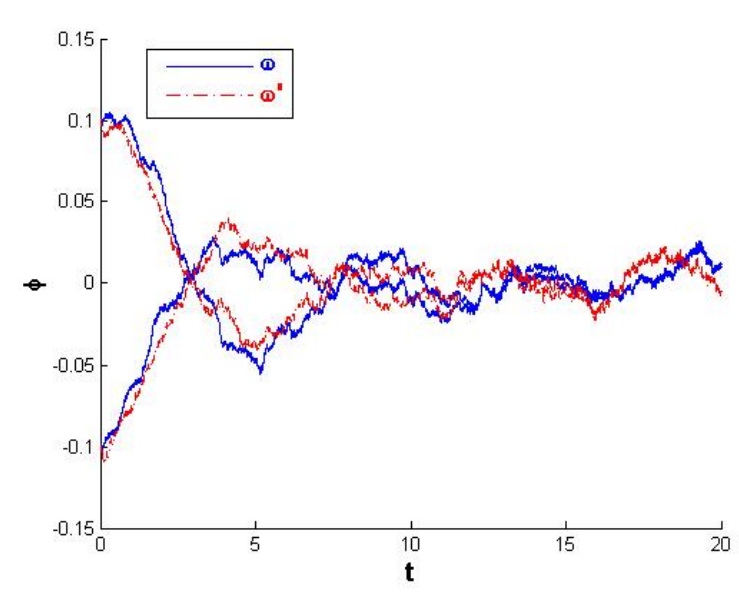

(a) $c=0.5$

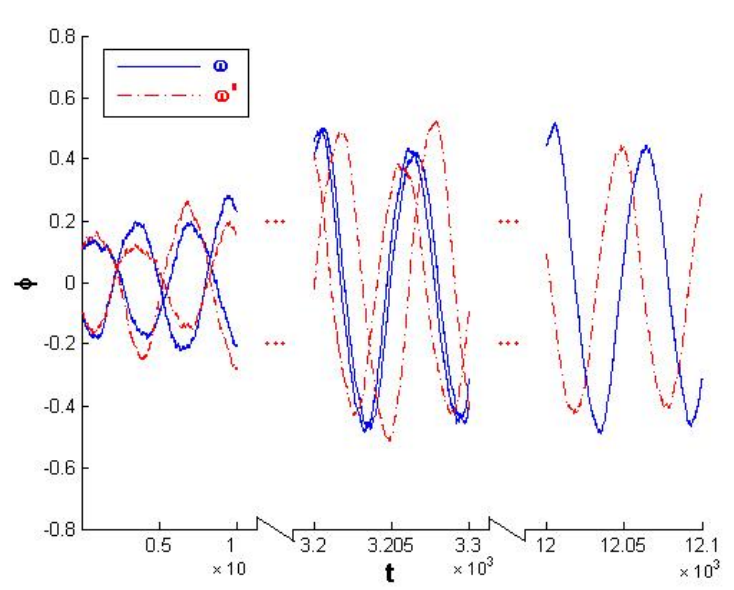

(b) $c=1.1$

Figure 2: Convergence process of $\varphi\left(t, \vartheta_{-t} \omega\right) \mathbf{x}_{0}$ when $a=1, \alpha=2, \beta=1, \sigma=0.02$ from different initial values and different orbits of the Wiener process $\left\{\omega_{t}\right\}$.

Fig. 2 displays sample paths for two different initial values and two orbits of the Wiener process, $\omega$ and $\omega^{\prime}$. As $t \rightarrow \infty$, Fig. 2 shows that the sample paths converge, which means the existence of a random fixed point which depends on the orbit of the Wiener process. In the case of $c=0.5$, there is no particular structure but in the case of $c=1.1$, the sample paths converge with some pattern. From Fig. 2, we can see the 


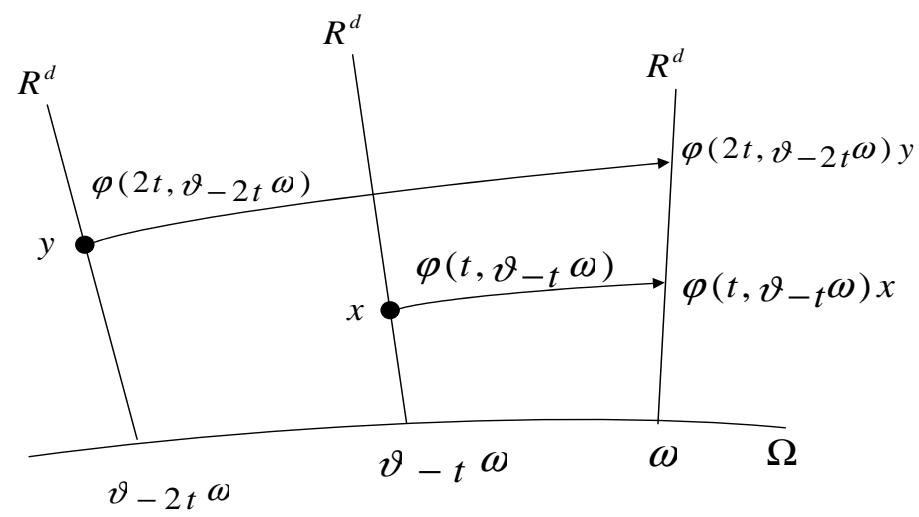

Figure 3: The pullback process $\varphi\left(t, \vartheta_{-t} \omega\right)$.

dynamical behaviour of the random fixed point, that is the random invariant measure, undergoes some change as the parameter $c$ varies.

To actually give more information about invariant measures, we still need to examine global random attractors. A global random attractor is a measurable map $\omega \rightarrow A(\omega)$ such that $A(\omega)$ is compact in $\mathbb{R}^{2}$, invariant (i.e. $\varphi(t, \omega) A(\omega)=A\left(\vartheta_{t} \omega\right)$ for any $t, \omega$ ), and it attracts any bounded deterministic set $D \subset \mathbb{R}^{2}$, that is $d\left(\varphi\left(t, \vartheta_{-t} \omega\right) D, A(\omega)\right) \rightarrow 0$ when $t \rightarrow \infty$, where $d\left(S_{1}, S_{2}\right):=\sup _{s_{1} \in S_{1}} \inf \left\{d\left(s_{1}, s_{2}\right), s_{2} \in S_{2}\right\}$. Random attractors are of particular importance since on them the long-term behaviour of the system takes place. In addition, global random attractors are connected and they support all invariant measures (see Crauel and Flandoli [7] and Crauel [8]).

In the definition of a random attractor, a pullback process $\varphi\left(t, \vartheta_{-t} \omega\right)$ is used, which is illustrated in Fig. 3. Note that the definition of a global random attractor is based on following the evolution of a whole set of initial points from time $-t$ to time 0 (and not from 0 to $t$ ). This enables us to study the asymptotic behaviour as $t \rightarrow \infty$ in the fixed fibre at time 0 . By increasing $t$ the mapping is made to start at successively earlier times, corresponding to a pull back in time. The results from the pullback operation are shown in Fig. 4 with different initial values and different parameters $c$ at different times $t$. As in the calculation of the Lyapunov exponents, we fix the parameters $a=1, \alpha=2$, $\beta=1, \sigma=0.02$, choose two different values of $c, c=0.5$ and 1.1 , and use a uniform distribution as the initial value set $D$.

When $c=0.5$, through the pullback process, we can see from Fig. 4(a) that $\varphi_{c}\left(t, \vartheta_{-t} \omega\right) D$ shrinks to a random fixed point $\mathbf{x}^{*}(\omega)$ which is distinct from zero. This is because the small additive noise perturbs the invariance of the zero fixed point of the deterministic system. Moreover, numerically it can be observed that, under time reversion, the solution of the system satisfies $\varphi_{c}\left(-t, \vartheta_{t} \omega\right) \mathbf{x}_{0} \rightarrow \infty(t \rightarrow \infty)$ for any $\mathbf{x}_{0} \neq \mathbf{x}^{*}(\omega)$, which implies that there is no other invariant measure. Linking with the calculation of the Lyapunov exponents in Fig. 1, we know that the system is stable at this value of $c$ because of the negative largest Lyapunov exponents, and the system has a unique and stable invariant measure which is a random Dirac measure $\mu_{\omega}=\delta_{\mathbf{x}^{*}(\omega)}$ whilst the global 

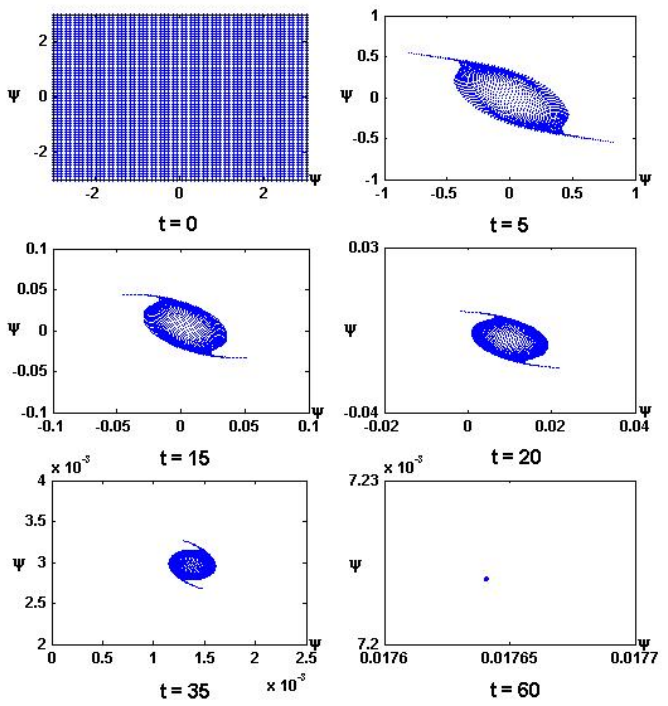

(a) $c=0.5$
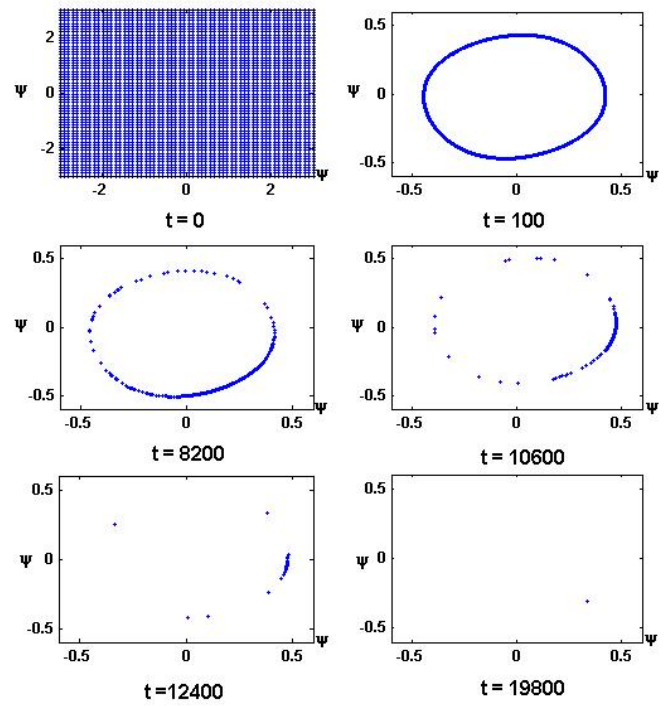

(b) $c=1.1$

Figure 4: Random attractors of $\varphi\left(t, \vartheta_{-t} \omega\right) D$ when $a=1, \alpha=2, \beta=1, \sigma=0.02$, where the initial value set $D$ comes from a uniform distribution.

random attractor is $A(\omega)=\left\{\mathbf{x}^{*}(\omega)\right\}$. This is exactly the stochastic analogue for the corresponding deterministic case discussed in the previous section.

When $c=1.1$, we observe from Fig. 1 the occurrence of positive Lyapunov exponents. Applying the pullback operation, we see from Fig. 4(b) that a different behaviour emerges, compared to the case of $c=0.5$, during the convergence of $\varphi_{c}\left(t, \vartheta_{-t} \omega\right) D$. A random circle becomes visible (at $t=100$ in Fig. 4(b)) and further convergence takes place on this circle. At last, $\varphi_{c}\left(t, \vartheta_{-t} \omega\right) D$ converges to a random point $\mathbf{x}^{\sharp}(\omega)$. We find that, again, the invariant measure is a random Dirac measure $\nu_{\omega}=\delta_{\mathbf{x}^{\sharp}(\omega)}$ which is stable with the nonpositive largest Lyapunov exponent. However, through the time reversion solution $\varphi_{c}\left(-t, \vartheta_{t} \omega\right) \mathbf{x}_{0}(t \rightarrow \infty)$, we show that the invariant measure $\mu_{\omega}=\delta_{\mathbf{x}^{*}(\omega)}$ exists in the interior of the circle, which is illustrated in Fig. 5. Also, the invariant measure $\mu_{\omega}=\delta_{\mathbf{x}^{*}(\omega)}$ is unstable and has two positive Lyapunov exponents. In addition, under time reversion, $\mathbf{x}^{\sharp}(\omega)$ is not attracting. As suggested in Schenk-Hoppé [14], another invariant measure, say $\nu_{\omega}^{\prime}$, on the random circle exists, see Fig. 5. This analysis implies that, for $c=1.1$, there exist more than two invariant measures, one is completely stable and one is completely unstable, and the global random attractor $A(\omega)$ which supports all invariant measures is a random disc whose boundary is a random circle shown in Fig. $4(\mathrm{~b})(\mathrm{t}=100)$.

In summary, our analysis on the D-bifurcation gives us insights into the significant impact of the chartists on the market equilibrium distributions. These distributions can be characterized by the invariant measures of the SDEs. We show that there exists a unique stable invariant measure in the market. However, the stable invariant measure changes quantitatively when the chartists change their extrapolation of the trend. The 


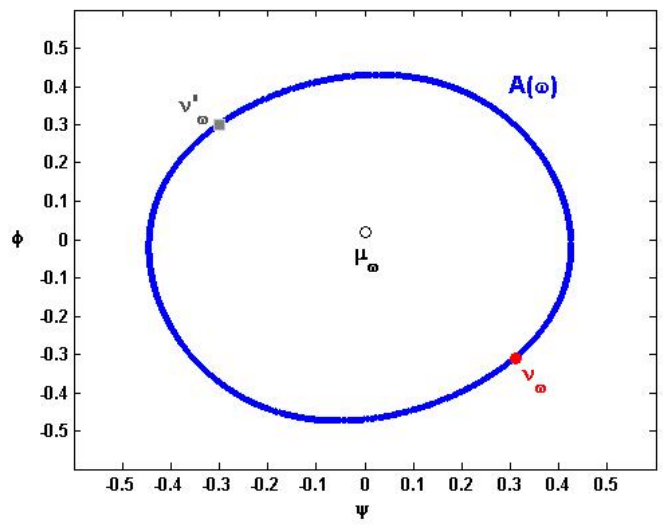

$\mu_{\omega}$ : unstable invariant measure (o)

$\nu_{\omega}:$ stable invariant measure $(\bullet)$

$\nu_{\omega}^{\prime}$ : additional invariant measure (-)

Figure 5: Global random attractor for $c=1.1, a=1, \alpha=2, \beta=1$ and $\sigma=0.02$.

change can be described by the stochastic Hopf bifurcation. We have observed that the Hopf bifurcation remains on the level of the invariant measures as the loss of stability of a measure and occurrence of a new stable measure, and on the level of the global attractor as the change from a random point to a random disc.

\section{P-Bifurcation}

The analysis of the D-bifurcation gives us a perspective from a dynamical systems viewpoint by focusing on the pathwise evolution of the random dynamical system. However with SDEs, there is also a statistical viewpoint. To illustrate the statistical characteristic of a random dynamical system, the stationary measure is an appropriate choice to describe the long term behaviour of solutions of differential equations with random perturbations. The Phenomenological $(P)$-bifurcation approach to stochastic bifurcation theory examines the qualitative changes of the stationary measures.

A probability measure $\rho$ on $\left(\mathbb{R}^{2}, \mathcal{B}\left(\mathbb{R}^{2}\right)\right)$ is called stationary if for all $t \geq 0, B \in \mathcal{B}\left(\mathbb{R}^{2}\right)$, $\int_{\mathbb{R}^{2}} P(t, \mathbf{x}, B) \rho(d \mathbf{x})=\rho(B)$, where $P(t, \mathbf{x}, B)=\mathbb{P}(\varphi(t, \omega) \mathbf{x} \in B)$ is generated by $\varphi$ for time $\mathbb{R}_{+}$. If $\rho$ has a density $\mathbb{p}$, the stationarity of $\rho$ is equivalent to the statement that $\mathbb{p}$ is a stationary (i.e. time independent) solution of the Fokker-Planck equation. The P-bifurcation approach studies qualitative changes of densities of stationary measures $\rho_{c}$ when a parameter, in this case $c$, varies. Hence, for the P-bifurcation, we are only interested in the changes of the shape of the stationary density. Thus we first use the Euler-Maruyama scheme and calculate one sample path up to time 500,000 with step size 0.001 . Since the average amount of time this solution path spends in each sample set is approximately equal to the measure of this set, we obtain a histogram as an estimation of the density of the stationary measure. For a better visualization, the density functions are then smoothed by using a standard procedure.

For $a=1, \alpha=2, \beta=1$, and $\sigma=0.02$, Fig. 6 shows qualitatively different joint and marginal stationary densities for the different $c$ values of $0.5,1$, and 1.1. Fig. 6 shows the joint stationary densities (the first panel) and the marginal densities (the second and 


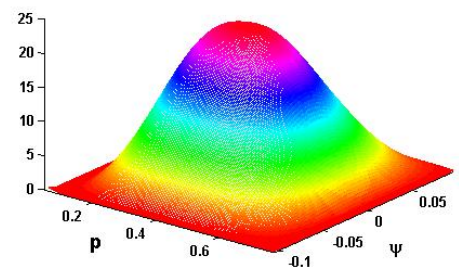

(a) $c=0.5$

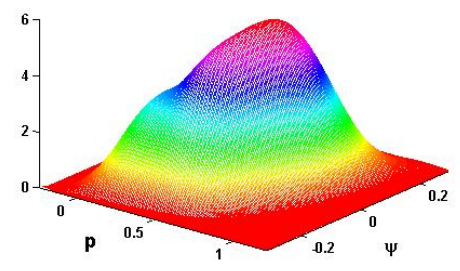

(d) $c=1.0$

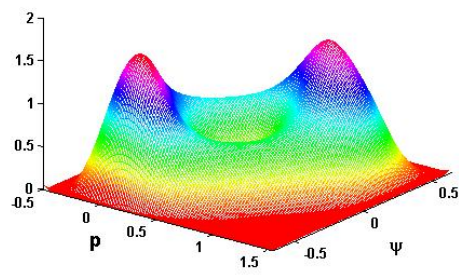

(g) $c=1.1$

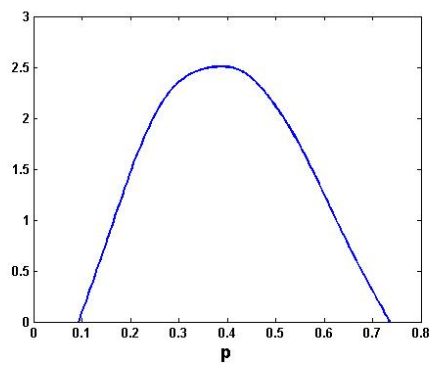

(b) $c=0.5$

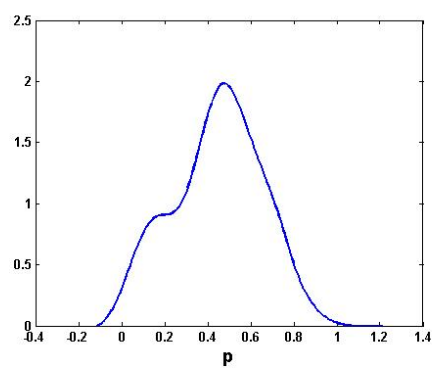

(e) $c=1.0$

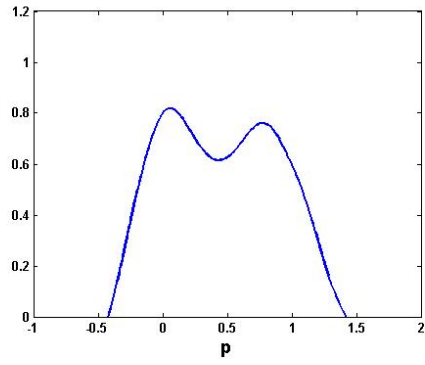

(h) $c=1.1$

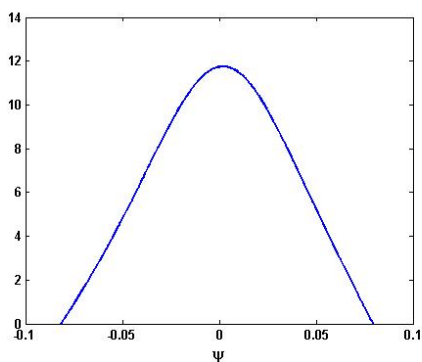

(c) $c=0.5$

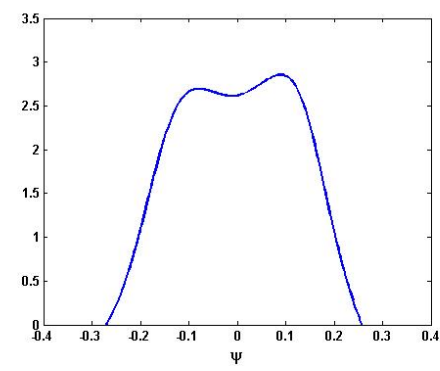

(f) $c=1.0$

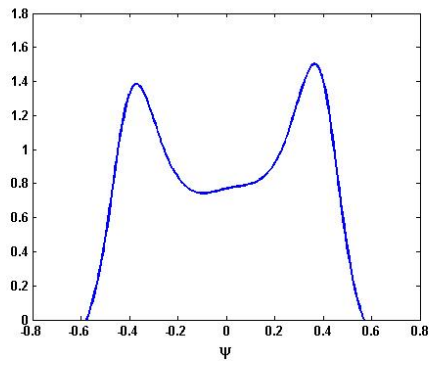

(i) $c=1.1$

Figure 6: Joint stationary densities of the price $p$ and the assessment of the price trend $\psi$ and the corresponding marginal distributions for $p$ and $\psi$-axis for $a=1, \alpha=2, \beta=1$, $\sigma=0.02$.

third panels) for the variables $p$ and $\psi$.

Note that there is a one-to-one correspondence between the stationary measure $\rho$ and the invariant measure $\mu_{\omega}$ which is measurable with respect to the past $\mathcal{F}_{-\infty}^{0}:=$ $\sigma\left(W_{s}, s \leq 0\right)$ (the $\sigma$-algebra generated by $\left\{W_{s}\right\}_{s \leq 0}$ ). This correspondence is given by $\mu_{\omega} \rightarrow \mathbb{E} \mu_{\omega}=\rho$ and $\rho \rightarrow \lim _{t \rightarrow \infty} \varphi\left(t, \vartheta_{-t} \omega\right) \rho:=\mu_{\omega}$ (see Arnold [1]). Then combining with the analysis of D-bifurcation, we can see from Fig. 6 that, for $c=0.5$, the joint densities in the $(p, \psi)$ planes have one peak and the marginal densities for either $\psi$ or $p$ are unimodal, which corresponds to the stable Dirac invariant measure $\mu_{\omega}=\delta_{\mathbf{x}^{*}}(\omega)$ with the global random attractor $A(\omega)=\left\{\mathrm{x}^{*}(\omega)\right\}$ under the D-bifurcation analysis. However, for $c=1.1$, the joint density in the $(p, \psi)$ plane has a crater-like shape and the marginal densities for either $\psi$ or $p$ are bimodal. This change is underlined by the stable Dirac invariant measure $\nu_{\omega}=\delta_{\mathbf{x}^{\sharp}}(\omega)$ with the global random attractor of a random disc under the D-bifurcation analysis. For $c=1$, the joint (marginal) density can be regarded as the transition from single peak to crater-like (from unimodal to bimodal) density. 
Therefore, as the chartists' adjustment parameter $c$ increases, the qualitative changes of the stationary density indicates the occurrence of a P-bifurcation.

\section{Conclusion}

This paper presents a continuous-time stochastic asset pricing model in a speculative financial market with fundamentalists and chartists. By applying stochastic bifurcation theory, we examine the limiting market equilibrium distribution numerically. We have shown that speculative behaviour of chartists can lead the market price to display different forms of equilibrium distributions. We have demonstrated the combined analysis of both D- and P-bifurcations certainly gives us a relatively complete picture of the stochastic behaviour of our model. In particular, when the chartists extrapolate the trend weakly (so that $c<c_{D}$ ), the system only has one invariant measure $\delta_{\mathbf{x}^{*}(\omega)}$ which is stable. In this case, $\mathbf{x}^{*}(\omega)$ has a stationary measure which has one peak. However, when the chartists extrapolate the trend strongly (so that $c>c_{D}$ ), a new stable random Dirac measure $\delta_{\mathbf{x}^{\sharp}(\omega)}$ appears and the corresponding stationary measure has a crater-like density. The change of stationary distribution is characterized by a bimodal logarithm price distribution and fat tails.

To conclude the paper, we refer to Chiarella, He and Zheng [6] for more detailed analysis of the stochastic bifurcation of the fundamentalist-chartist model and the relationship between the stochastic and deterministic dynamics of the model. The cited paper provides more insights into the model of stochastic behaviour through stochastic bifurcation analysis, stochastic approximation methods, and the difference between deterministic and stochastic dynamics. It demonstrates that stochastic bifurcation analysis can be a powerful tool to help in understanding various types of financial market behaviour.

\section{References}

[1] Arnold, L. Random Dynamical Systems, Springer, New York, 1998.

[2] Arnold, L., Sri Namachchivaya, N. and Schenk-Hoppé, K. R. Towards an understanding of stochastic Hopf bifurcation: a case study, Int. J. Bifurcat. Chaos 6 (1996) 1947-1975.

[3] Beja, A., Goldman, M.B. On the dynamic behaviour of prices in disequilibrium, J. Finance 35 (1980) 235-248.

[4] Böhm,V., Chiarella, C. Mean Variance Preferences, Expectations Formation, and the Dynamics of Random Asset Prices, Math. Finance 15 (2005) 61-97. 
[5] Chiarella, C. The dynamics of speculative behaviour, Ann. Oper. Res. 37 (1992) 101-123.

[6] Chiarella, C., He, X., Zheng, M. The Stochastic Price Dynamics of Speculative Behaviour, 2007, working paper, QFRC, Univerisy of Technology, Sydney.

[7] Crauel, H., Flandoli, F. Attractors for random dynamical systems, Probab. Theor. Relat. Field 100 (1994)365-393.

[8] Crauel, H. Invariant measures are supported by random attractors, 1995, preprint.

[9] Föllmer, H., Horst, U., Kirman, A. Equilibria in financial markets with heterogeneous agents: a probabilistic perspective, J. Math. Econ. 41 (2005) 123-155.

[10] Oseledets, V.I. A Multiplicative Ergodic Theorem. Lyapunov Characteristic Numbers for Dynamical Systems, Trans. Moscow Math. Soc. 19 (1968) 197-231.

[11] Parker, T.S., Chua, L.O. Practical Numerical Algorithms for Chaotic Systems, Springer-Verlag, New York, 1989.

[12] Rheinlaender, T., Steinkamp, M. A stochastic version of Zeeman's market model, Stud. Nonlinear Dynam. Econometrics 8 (2004) 1-23.

[13] Schenk-Hoppé, K. R. Deterministic and stochastic Duffing-van der Pol oscillators are non-explosive, Z. Angew. Math. Phys. 47 (1996) 740-759.

[14] Schenk-Hoppé, K. R. Bifurcation Scenarios of the noisy Duffing-van der Pol oscillator, Nonlinear Dynam. 11 (1996) 255-274.

[15] Zeeman, E. The unstable behavior of stock exchanges, J. Math. Econ. 1 (1974) 39-49. 